\title{
SUBSTITUTIONS PAR DES MOTIFS EN DIMENSION 1*
}

\author{
N. Pytheas FogG ${ }^{1}$
}

\begin{abstract}
Pattern subsitutions in dimension 1. A substitution is a morphism of the free monoid: each letter is mapped to a word, and the image of a word is the concatenation of the images of its letters. This paper introduces a generalization of the notion of substitution, where the image of a letter is not a word but a pattern, i.e., a "word with holes": the image of a word is obtained by connecting the patterns corresponding to each of the letters by means of local rules. We completely characterize pattern substitutions which are defined on every biinfinite sequence, and we explain how to build them. We show that every biinfinite sequence which is a fixed point of a pattern substitution is substitutive, i.e., it is the image, by a letter to letter morphism, of a fixed point of a substitution (in the usual meaning).
\end{abstract}

Résumé. Une substitution est un morphisme de monoïdes libres : chaque lettre a pour image un mot, et l'image d'un mot est la concaténation des images de ses lettres. Cet article introduit une généralisation de la notion de substitution, où l'image d'une lettre n'est plus un mot mais un motif, c'est-à-dire un « mot à trous », l'image d'un mot étant obtenue en raccordant les motifs correspondant à chacune de ses lettres à l'aide de règles locales. On caractérise complètement les substitutions par des motifs qui sont définies sur toute suite biinfinie, et on explique comment les construire. On montre que toute suite biinfinie qui est point fixe d'une substitution par des motifs est substitutive, c'est-à-dire est l'image, par un morphisme lettre à lettre, d'un point fixe de substitution (au sens usuel).

Classification Mathématique. 68R15, 37B10.

\footnotetext{
Mots Clés. Substitutions, mots, motifs, pavages de la droite, combinatoire des mots. Substitutions, words, patterns, tilings of the line, word combinatorics.

* Cet article a été rédigé grâce à la contribution de P. Arnoux, V. Berthé et J. Cassaigne.

${ }^{1}$ Institut de mathématiques de Luminy, CNRS UMR 6206 / FRUMAM, case 907, 163 avenue de Luminy, 13288 Marseille Cedex 9, France; pytheas@iml.univ-mrs.fr
}

(C) EDP Sciences 2007 


\section{INTRODUCTION}

Les substitutions sont des objets combinatoires naturels qui engendrent des suites infinies par itération, en remplaçant une lettre par un mot; une substitution n'est en fait rien d'autre qu'une « macro ». Une des caractéristiques les plus intéressantes des suites substitutives est qu'elles présentent une structure autosimilaire très ordonnée, tout en étant engendrées par un procédé algorithmique élémentaire, et ce sans être périodiques en général. Les suites substitutives et les systèmes dynamiques engendrés par substitutions entretiennent de nombreuses interactions avec, entre autres domaines, la combinatoire des mots et l'informatique théorique [14,15], la physique [2], la théorie ergodique et spectrale [18,19], la géométrie des pavages [8,13,25], les systèmes de numération $[7,26]$, l'approximation diophantienne [21,22], ou les problèmes de transcendance $[1,2]$.

Plus précisément, une substitution $\sigma$ est un morphisme de monoïdes libres $\sigma: \mathcal{A}^{*} \rightarrow \mathcal{B}^{*}$. Si $\sigma$ est non-effaçante, c'est-à-dire si elle envoie un mot non vide sur un mot non vide, elle s'étend aux suites infinies ou biinfinies de $\mathcal{A}^{\mathbb{N}}$ et $\mathcal{A}^{\mathbb{Z}}$, respectivement. De plus, quand $\mathcal{B}=\mathcal{A}, \sigma$ est non effaçante et $\sigma(a) \in a \mathcal{A}^{+}$, on peut alors itérer $\sigma$ et définir le mot infini $\sigma^{\infty}(a)=\lim _{k \rightarrow \infty} \sigma^{k}(a) \in \mathcal{A}^{\mathbb{N}}$ qui est un point fixe de $\sigma$.

On construit ainsi deux classes de mots infinis (pour plus de détails, voir par exemple $[2,14,18,20])$ :

- les points fixes de substitutions, ou mots DOL; des exemples classiques en sont les suites de Thue-Morse ou de Fibonacci;

- les mots substitutifs, ou mots CDOL, qui sont les images des premiers par une autre substitution (que l'on peut toujours supposer lettre à lettre, c'est-à-dire que l'image de toute lettre est encore une lettre) [2]; des exemples classiques en sont les suites de pliage de papier ou de Rudin-Shapiro.

Le but de cet article est d'étendre la définition de substitution pour associer à chaque lettre un mot non nécessairement connexe (donc éventuellement à trous), que nous appellerons motif pour éviter toute confusion alors que mot désignera comme usuellement un motif connexe. Les substitutions entretenant d'étroites relations avec les pavages et la numération, il paraît en effet relativement naturel de tenter d'étendre la combinatoire des mots sous-jacente à des motifs. Une tentative de définition de la notion de complexité (qui compte le nombre de facteurs de longueur donnée d'une suite), à savoir la complexité en motifs, a été ainsi développée dans $[11,12]$.

Nous introduisons donc une notion de «substitutions à trous » associant à une lettre non pas un mot mais un motif. Il nous manque néanmoins une information pour savoir comment appliquer la substitution à un mot : nous savons comment remplacer une lettre mais nous ne savons pas a priori où disposer son image. Quand nous travaillons avec des mots, il suffit d'utiliser l'ordre naturel de $\mathbb{N}$ ou $\mathbb{Z}$, ou d'utiliser la structure de monoïde pour la concaténation. Nous allons donc introduire plusieurs conditions nous permettant d'obtenir l'image d'un mot. Une 
question se pose de plus naturellement : comment et sous quelles conditions peuton étendre le domaine de définition d'une telle substitution à tout $\mathcal{A}^{\mathbb{Z}}$ afin qu'elle soit définie partout et sans ambiguïté? Il faut pour cela faire en sorte que les supports de tous les motifs correspondant aux images des lettres forment une partition de $\mathbb{Z}$; on est donc ramené à un problème de pavage de $\mathbb{Z}$. Nous allons ainsi imposer les conditions suivantes :

- les substitutions par des motifs s'étendent à toutes les suites biinfinies, et les envoient sur des suites biinfinies;

- le placement de l'image d'une lettre ne dépend que du voisinage de cette lettre; autrement dit, on cherche des règles locales telles que, pour toute suite biifinie $u \in \mathcal{A}^{\mathbb{Z}}$, les supports des occurrences des images des lettres de $u$, décalées en utilisant les règles locales, forment une partition de $\mathbb{Z}$.

Cette définition impose alors des conditions très restrictives. Notons que la notion de règles locales est inspirée par la notion de pavages substitutifs : dans ce contexte, les substitutions produisent des règles d'agencement agissant sur un ensemble fini de prototuiles et déterminent la manière dont les tuiles vont s'assembler (voir par exemple $[8,25]$ ). L'exemple le plus connu en est le pavage de Penrose. Nous déterminons complètement les substitutions unidimensionnelles qui sont partout définies (théorème 3.6) et montrons que leurs points fixes sont images par un morphisme lettre à lettre d'un point fixe de substitution au sens usuel (théorème 4.1).

Ce dernier résultat indique qu'il faut passer en dimension deux, au moins, pour voir des phénomènes réellement nouveaux en termes de substitutions. Notons que l'une des motivations à ce travail provient d'ailleurs du cadre multidimensionnel. Comment généraliser la notion de substitution à plusieurs dimensions? On peut substituer à chaque lettre un mot rectangulaire, mais cela ne fonctionne que si tous les rectangles ont des formes adaptées (voir par exemple [10,16] ou [17]); ce sont en particulier des substitutions uniformes si tous les rectangles ont la même forme, qui correspondent à une généralisation de la notion de suite automatique [2, 9, 23, 24]. On peut aussi utiliser d'autres formes, non nécessairement connexes [3-5].

Cet article est organisé comme suit : nous introduisons au paragraphe 2 le formalisme nécessaire à l'introduction de notre notion de substitution par des motifs. Rappelons que nous voulons ici que nos substitutions puissent s'appliquer à toute suite biinfinie sans chevauchement éventuel des images des lettres; nous introduisons ainsi une notion de substitution par des motifs avec règles locales. Nous donnons un théorème de caractérisation au paragraphe 3 et montrons au paragraphe 4 que tout point fixe d'une substitution par des motifs avec règles locales est substitutif. Enfin nous concluons au paragraphe 5 par quelques perspectives et une discussion du cas multidimensionnel, qui a motivé ce travail.

\section{DÉfinitions et notations}

\subsection{Mots ET MOTIFS}

On considère un alphabet fini $\mathcal{A}$. 
Définition 2.1. Un motif pointé d'alphabet $\mathcal{A}$ est une application $u$ d'une partie finie $S$ de $\mathbb{Z}$ dans $\mathcal{A}$. On appelle support de $u$, noté Supp $u$, l'ensemble $S \subset \mathbb{Z}$. Le poids de $u$, que l'on note $\omega(u)$, est défini comme le cardinal $|S|$ de $S$, et le nombre $l(u)=\max S-\min S+1$ est la longueur de $u$ (avec par convention $l(u)=0$ si Supp $u=\emptyset)$.

L'ensemble $\mathbb{Z}$ agit naturellement sur l'ensemble des motifs pointés par translation : étant donné un motif pointé $u$ de support $S$, le motif pointé $v=u+n$ est le motif dont le support est $S+n=\{i+n \mid i \in S\}$, et tel que $v_{i+n}=u_{i}$ pour $i \in S$.

Si $u$ et $v$ sont deux motifs pointés de supports disjoints, on note $u \cup v$ le motif pointé de support $\operatorname{Supp} u \cup \operatorname{Supp} v$ qui cö̈ncide avec $u$ et $v$ sur leurs supports respectifs. De même, on dit que $u$ est facteur de $v$ et on note $u \subset v$ si Supp $u \subset$ Supp $v$ et $u$ est égal à la restriction de $v$ à Supp $u$.

Définition 2.2. Un motif $U$ est une classe d'équivalence de motifs pointés sous l'action des translations. Le motif vide est le motif dont l'unique représentant est le motif pointé de support vide.

Définition 2.3. Le représentant canonique d'un motif non vide $U$ est le motif pointé $u \in U$ tel que min $\operatorname{Supp} u=0$.

On identifiera souvent un motif et son représentant canonique; en particulier, par convention, le support d'un motif est le support de son représentant canonique. Avec ces définitions, un mot (fini), au sens usuel, est un motif dont le support est un intervalle. Un motif pointé dont le support est un intervalle est appelé mot pointé.

On définit respectivement le poids $\omega(U)$ et la longueur $l(U)$ du motif $U$ comme les poids et longueur d'un de ses représentants (ces notions sont évidemment invariantes par translation).

Exemple 1. Nous noterons $U=a \sqcup b c \sqcup \sqcup b$ le motif dont le représentant canonique $u$ a pour support $\{0,2,3,6\}$ avec $u_{0}=a, u_{2}=b, u_{3}=c$ et $u_{6}=b$. Son poids est $\omega(U)=4$ et sa longueur $l(U)=7$.

On pose $\mathbb{N}=\{n \in \mathbb{Z} \mid n \geq 0\}, \mathbb{Z}^{+}=\{n \in \mathbb{Z} \mid n>0\}, \mathbb{Z}^{-}=\{n \in \mathbb{Z} \mid n<0\}$. Avec ces notations, $a^{\mathbb{Z}}$ désigne la suite constante de valeur $a$, et $a^{\mathbb{Z}^{-}} \cdot b^{\mathbb{N}}$ la suite $\left(u_{n}\right)_{n \in \mathbb{Z}}$ telle que $u_{n}=a$ si $n<0$ et $u_{n}=b$ si $n \geq 0$; le point placé entre $a^{\mathbb{Z}^{-}}$et $b^{\mathbb{N}}$ sert à indiquer la position de l'indice 0 (la lettre d'indice 0 se trouve immédiatement à la droite de ce point); $a^{\mathbb{Z}^{-}} \cdot b a^{\mathbb{Z}^{+}}$désigne donc la suite qui vaut $b$ en 0 et $a$ partout ailleurs.

\subsection{Germes de substitutions}

Nous introduisons ici une notion de substitution qui envoie les lettres sur des motifs pointés; il serait a priori plus naturel d'envoyer les lettres sur des motifs non pointés mais ce pointage se révèlera être utile au paragraphe 4 afin d'introduire la notion de suite biinfinie point fixe engendré par la substitution. 
Définition 2.4. Un germe de substitution est une application $\sigma$ d'un alphabet $\mathcal{A}$ dans l'ensemble des motifs pointés non vides d'alphabet $\mathcal{B}$ (éventuellement identique au premier). Un germe de substitution est dit canonique si les images des lettres coïncident avec le représentant canonique de leur classe. Il est dit uniforme si les images des lettres ont toutes le même support.

Définition 2.5. Si $\sigma$ est un germe de substitution, le germe canonique de substitution associé à $\sigma$, noté $\sigma_{\mathrm{c}}$, est le germe canonique tel que, pour toute lettre $a \in \mathcal{A}$, $\sigma_{\mathrm{c}}(a)$ est le représentant canonique de la classe de $\sigma(a)$.

La plupart des propriétés de $\sigma$ se transposent sur $\sigma_{\mathrm{c}}$ (voir lemme 2.12), pour lequel elles s'expriment plus simplement. Aussi, dans la suite de ce paragraphe et au paragraphe 3 , nous considèrerons le plus souvent des germes canoniques de substitutions afin de simplifier le formalisme.

Une question se pose naturellement : comment et sous quelles conditions peuton, à partir d'un germe de substitution $\sigma$, construire une application de $\mathcal{A}^{\mathbb{Z}}$ dans $\mathcal{B}^{\mathbb{Z}}$, définie partout et sans ambiguïté? Il faut pour cela faire correspondre à chaque occurence d'une lettre $a$ un motif pointé équivalent à $\sigma(a)$, de telle façon que les supports de tous ces motifs forment une partition de $\mathbb{Z}$, d'où la définition suivante :

Définition 2.6. Soit $x=\left(x_{n}\right)_{n \in \mathbb{Z}} \in \mathcal{A}^{\mathbb{Z}}$ et $\sigma$ un germe de substitution. Une réalisation de $\sigma$ sur $x$ est la donnée d'une famille d'entiers $t=\left(t_{n}\right)_{n \in \mathbb{Z}}$ telle que

$$
\left\{\operatorname{Supp} \sigma\left(x_{n}\right)+t_{n} \mid n \in \mathbb{Z}\right\}
$$

forme une partition de $\mathbb{Z}$.

L'image de la suite $x$ par $\sigma$ selon $t$ est alors la suite $\operatorname{Im}(\sigma, t)(x)=y=\left(y_{n}\right)_{n \in \mathbb{Z}} \in \mathcal{B}^{\mathbb{Z}}$ définie par

pour tous $n \in \mathbb{Z}$ et $k \in \operatorname{Supp} \sigma\left(x_{n}\right)$.

$$
y_{t_{n}+k}=\sigma\left(x_{n}\right)_{k}
$$

En étendant à une famille infinie la notion d'union de deux motifs, on peut noter $\operatorname{Im}(\sigma, t)(x)=\bigcup_{n \in \mathbb{Z}} \sigma\left(x_{n}\right)+t_{n}$, et dire que le motif $\sigma\left(x_{n}\right)+t_{n}$ est facteur de $\operatorname{Im}(\sigma, t)(x)$.

Exemple 2. Considérons un germe canonique de substitution $\sigma$ sur l'alphabet $\{a\}$ (mais dont les images sont des motifs d'alphabet $\{a, b\}$ ) tel que l'image de la lettre $a$ soit $\sigma(a)=a \sqcup \sqcup b$. Une réalisation possible de $\sigma$ sur la suite $x=a^{\mathbb{Z}}$ est donnée par la suite $t=(2 n)_{n \in \mathbb{Z}}$, ce qui donne pour image la suite périodique

$$
\operatorname{Im}(\sigma, t)(u)=\ldots a b a b a b a b \cdot a b a b a b a b \ldots .
$$

Une autre réalisation est donnée par la suite

$$
t^{\prime}=(\ldots,-6,-5,-4,0,1,2,6,7,8, \ldots)=\left(n+3\left\lfloor\frac{n}{3}\right\rfloor\right)_{n \in \mathbb{Z}},
$$

ce qui donne

$$
\operatorname{Im}\left(\sigma, t^{\prime}\right)(u)=\ldots \text { bbbaaabbb·aaabbbaaa } \ldots
$$


La définition n'impose pas qu'une réalisation soit une suite croissante : ici, comme $x=a^{\mathbb{Z}}$, n'importe quelle permutation de $t$ ou de $t^{\prime}$ convient aussi, et ce sont d'ailleurs les seules réalisations possibles, à translation près.

Notons qu'il existe des germes de substitutions qui n'ont pas de réalisation; il suffit par exemple de considérer, sur l'alphabet $\{a\}$, le germe de substitution donné par $\sigma(a)=a a \sqcup a a$. Il existe aussi des germes de substitutions qui n'admettent des réalisations que sur certaines suites; par exemple, le germe de substitution $\sigma$ sur l'alphabet $\{a, b\}$ défini par $\sigma(a)=a \sqcup a a \sqcup a$ et $\sigma(b)=b b b$ admet une réalisation sur les suites constantes $a^{\mathbb{Z}}$ et $b^{\mathbb{Z}}$, mais pas sur la suite périodique ...abab.abab...

\subsection{RÈGLES LOCALES}

Si l'on suppose maintenant que la réalisation d'un germe est invariante par translation (c'est-à-dire que les images de deux suites biinfinies décalées l'une de l'autre sont elles-même décalées) et qu'elle ne dépend que du voisinage d'une lettre, on arrive à une définition bien plus restrictive :

Définition 2.7. On appelle règles locales pour l'alphabet $\mathcal{A}$ une matrice $D=$ $\left(D_{a b}\right)_{a, b \in \mathcal{A}}$ à coefficients dans $\mathbb{Z}$.

Définition 2.8. Soient un germe de substitution $\sigma$ défini sur $\mathcal{A}$, des règles locales $D$ pour $\mathcal{A}$, un mot $x=\left(x_{n}\right) \in \mathcal{A}^{\mathbb{Z}}$, et la suite d'entiers $t=\left(t_{n}\right)_{n \in \mathbb{Z}}$ définie par $t_{0}=0$ et $t_{n+1}-t_{n}=D_{x_{n} x_{n+1}}$ pour tout $n \in \mathbb{Z}$. Si $t$ est une réalisation de $\sigma$ sur $x$, on dit que $t$ est conforme $\grave{a} D$.

Définition 2.9. Une substitution par des motifs définie par des règles locales est la donnée

- d'un germe de substitution $\sigma$, défini sur $\mathcal{A}$ et à valeurs sur $\mathcal{B}$,

- de règles locales $D$ pour $\mathcal{A}$,

tels que $\sigma$ admette sur tout mot $x \in \mathcal{A}^{\mathbb{Z}}$ une réalisation conforme à $D$.

On dit alors que les règles locales $D$ font du germe de substitution $\sigma$ une substitution. Cette substitution, que l'on notera $\sigma_{D}$, induit une application $\sigma_{D}: \mathcal{A}^{\mathbb{Z}} \rightarrow \mathcal{B}^{\mathbb{Z}}$, définie par $\sigma_{D}(x)=\operatorname{Im}(\sigma, t)(x)$, où $t$ est la réalisation de $\sigma$ sur $x$ conforme à $D$. Si le germe de substitution $\sigma$ est canonique, la substitution $\sigma_{D}$ est également dite $c a$ nonique. Si $\sigma$ est uniforme et si les entiers $D_{a b}$ sont tous égaux, la substitution $\sigma_{D}$ est dite uniforme.

Exemple 3. En reprenant l'exemple 2, avec $\sigma(a)=a \sqcup \sqcup b$, on voit facilement que la règle locale $D_{a a}=2$ nous donne une substitution canonique uniforme définie par des règles locales.

Définition 2.10. Les règles locales $\left(D_{a b}\right)_{a, b \in \mathcal{A}}$ sont dites croissantes si elles satisfont $D_{a a}>0$ pour tout $a \in \mathcal{A}$, et décroissantes si $D_{a a}<0$ pour tout $a \in \mathcal{A}$.

Lemme 2.11. Soit $\sigma$ un germe de substitution, et $D=\left(D_{a b}\right)_{a, b \in \mathcal{A}}$ des règles locales qui font de $\sigma$ une substitution. Alors

(i) les règles locales $D$ sont soit croissantes, soit décroissantes; 
(ii) les règles locales $D^{\prime}=-{ }^{t} D$, soit $D_{a b}^{\prime}=-D_{b a}$, font aussi de $\sigma$ une substitution; elles sont croissantes si les règles locales $D$ sont décroissantes, et inversement.

Preuve. Supposons d'abord que les règles locales $D$ ne sont ni croissantes, ni décroissantes : on peut donc trouver deux lettres $a$ et $b$ telles que $D_{a a} \geq 0$ et $D_{b b} \leq 0$. Considérons alors une réalisation $t$ de $\sigma$ sur $y=a^{\mathbb{Z}^{-}} \cdot a b^{\mathbb{Z}^{+}}$conforme aux règles locales $D$. La définition donne $t_{n}=n D_{a a}$ pour $n \leq 0$ et $t_{n}=D_{a b}+(n-1) D_{b b}$ pour $n>0$, de sorte que $t_{n} \leq \max \left(0, D_{a b}\right)$ pour tout $n \in \mathbb{Z}$. Mais si $t$ est majorée, les ensembles Supp $\sigma\left(y_{n}\right)+t_{n}$ ne peuvent pas former une partition de $\mathbb{Z}$.

Considérons maintenant les règles locales $D^{\prime}=-{ }^{t} D$, et une suite quelconque $x \in \mathcal{A}^{\mathbb{Z}}$. Soit $x^{\prime}$ l'image miroir de $x$, c'est-à-dire la suite $x^{\prime}=\left(x_{n}^{\prime}\right)_{n \in \mathbb{Z}}$ définie par $x_{n}^{\prime}=x_{-n}$. Par hypothèse, il existe une réalisation $t$ de $\sigma$ sur $x^{\prime}$ conforme aux règles locales $D$. Soit la famille d'entiers $t^{\prime}=\left(t_{n}^{\prime}\right)_{n \in \mathbb{Z}}$ définie par $t_{n}^{\prime}=t_{-n}$. On vérifie aisément que $t^{\prime}$ est une réalisation de $\sigma$ sur $x$ conforme aux règles locales $D^{\prime}$. Les règles locales $D^{\prime}$ font donc elles aussi de $\sigma$ une substitution, et on a $\sigma_{D^{\prime}}(x)=\sigma_{D}\left(x^{\prime}\right)$. Comme $D_{a a}^{\prime}=-D_{a a}$, il est clair que les règles locales $D^{\prime}$ sont croissantes si et seulement si les règles locales $D$ sont décroissantes.

Le lemme 2.11 nous autorise à ne considérer que des règles locales croissantes, ce que nous ferons dans toute la suite.

Lemme 2.12. Soit $\sigma$ un germe de substitution, et $\sigma_{\mathrm{c}}$ le germe canonique associé. Les règles locales $D=\left(D_{a b}\right)_{a, b \in \mathcal{A}}$ font de $\sigma$ une substitution si et seulement si les règles locales $D^{\prime}=\left(D_{a b}^{\prime}\right)_{a, b \in \mathcal{A}}$ font de $\sigma_{\mathrm{c}}$ une substitution, avec $D_{a b}^{\prime}=D_{a b}-$ $\min \operatorname{Supp} \sigma(a)+\min \operatorname{Supp} \sigma(b)$.

Preuve. Il suffit de voir que $\left(t_{n}\right)_{n \in \mathbb{Z}}$ est une réalisation de $\sigma$ sur une suite $x=$ $\left(x_{n}\right)_{n \in \mathbb{Z}}$ si et seulement si $\left(t_{n}^{\prime}\right)_{n \in \mathbb{Z}}$, avec $t_{n}^{\prime}=t_{n}+\min$ Supp $\sigma\left(x_{n}\right)$ est une réalisation de $\sigma_{\mathrm{c}} \operatorname{sur} x$, et qu'alors on a bien $t_{n+1}^{\prime}-t_{n}^{\prime}=D_{x_{n} x_{n+1}}^{\prime}$.

Nous pouvons maintenant déduire de la définition 2.8 une première condition nécessaire sur les règles locales dont on peut munir un germe canonique pour en faire une substitution.

Lemme 2.13. Toute substitution canonique par des motifs $\sigma$ définie par des règles locales croissantes $\left(D_{a b}\right)_{a, b \in \mathcal{A}}$ satisfait $D_{a b}=\omega_{a}$ pour tout couple de lettres $(a, b) \in$ $\mathcal{A}^{2}$, où $\omega_{a}=\omega(\sigma(a))$ désigne le poids de $\sigma(a)$.

Preuve. Soit $a$ une lettre fixée de $\mathcal{A}$. On note $l_{a}=l(\sigma(a))$ et $S_{a}=\operatorname{Supp} \sigma(a)$. Considérons la réalisation $t$ de $\sigma$ sur $x=a^{\mathbb{Z}}$ conforme aux règles locales : on a nécessairement $t_{n}=n D_{a a}$, pour $n \in \mathbb{Z}$, d'après la définition 2.8 , et $\left\{S_{a}+t_{n} \mid n \in \mathbb{Z}\right\}$ est une partition de $\mathbb{Z}$. Fixons $n \in \mathbb{N}$ et posons $E_{1}=\bigcup_{k<0}\left(S_{a}+t_{k}\right), E_{2}=$ $\bigcup_{0<k<n}\left(S_{a}+t_{k}\right)$ et $E_{3}=\bigcup_{k \geq n}\left(S_{a}+t_{k}\right)$, de sorte que $\left\{E_{1}, E_{2}, E_{3}\right\}$ est une partition de $\mathbb{Z}$. L'ensemble $E_{2}$ a exactement $n \omega_{a}$ éléments, et il est contenu dans l'intervalle $\left[0,(n-1) D_{a a}+l_{a}-1\right]$, puisque $S_{a} \subset\left[0, l_{a}-1\right]$. De même, $\left.\left.E_{1} \subset\right]-\infty,-D_{a a}+l_{a}-1\right]$ et $E_{3} \subset\left[n D_{a a},+\infty\right.$ [, donc $E_{2}$ contient l'intervalle $\left[l_{a}-D_{a a}, n D_{a a}-1\right]$. On en déduit les inégalités $(n+1) D_{a a}-l_{a} \leq n \omega_{a} \leq(n-1) D_{a a}+l_{a}$; comme ces inégalités sont vraies pour tout $n$, on doit avoir $D_{a a}=\omega_{a}$. 
Montrons maintenant que $D_{a b}=\omega_{a}$ pour toute lettre $b$ de $\mathcal{A}$. Par définition, les réalisations $\left(t_{n}\right)$ et $\left(t_{n}^{\prime}\right)$ des mots $x=a^{\mathbb{Z}}$ et $y=a^{\mathbb{Z}^{-}} \cdot a b^{\mathbb{Z}^{+}}$, respectivement, coïncident pour $n \leq 0$, tandis que pour $n>0, t_{n}=n D_{a a}$ et $t_{n}^{\prime}=D_{a b}+(n-1) D_{b b}$. On a alors

$$
\bigcup_{n \in \mathbb{Z}^{+}} S_{a}+t_{n}=\bigcup_{n \in \mathbb{Z}^{+}} S_{b}+t_{n}^{\prime}
$$

et donc, comme $\left(t_{n}\right)$ et $\left(t_{n}^{\prime}\right)$ sont strictement croissantes, $\min S_{a}+t_{1}=\min S_{b}+t_{1}^{\prime}$. De plus, $\sigma$ est canonique donc $\min S_{a}=\min S_{b}=0$, et il reste $t_{1}^{\prime}=t_{1}$, c'est-à-dire $D_{a b}=D_{a a}=\omega_{a}$.

Étant donné un germe de substitution $\sigma$, le lemme 2.13 garantit qu'il existe au plus un choix de règles locales croissantes $D$ qui font de $\sigma$ une substitution. Aussi, nous dénoterons désormais cette substitution $\sigma$ plutôt que $\sigma_{D}$.

Si $\sigma$ est une substitution définie par des règles locales, on peut définir l'image de $u$ dès que $u$ est un mot pointé dont le support contient 0 ; en effet, on associe à la lettre pointée $u_{0}$ de support $\{0\}$ le motif pointé image de $u_{0}$ par le germe $\sigma$; la position des images des autres lettres de $u$ est alors déterminée par les règles locales. Nous dénoterons $\sigma(u)$ l'image obtenue; notons que c'est un motif pointé. On peut également définir $\sigma(U)$, quand $U$ est un mot (non pointé), comme la classe de $\sigma(u)$, pour $u \in U ; \sigma(U)$ est alors un motif (non pointé). Nous avons donc étendu le domaine de définition de $\sigma$ aux mots (non pointés, ou pointés à condition que leur support contienne 0) et aux suites biinfinies. En général, il ne s'étend pas aux motifs, car la présence de trous empêche d'appliquer les règles locales; ce n'est possible que pour les substitutions uniformes.

Dans le cas d'une substitution classique (définie par des mots sans trous), on voit que le représentant canonique de tout mot $u$ est défini sur l'intervalle $[0,|u|-1]$; il suffit donc de poser $D_{a b}=|\sigma(a)|$ pour toute paire de lettres, et d'associer aux lettres le représentant canonique de leurs images selon cette substitution, pour retrouver la définition usuelle.

Remarque 1. La dissymétrie de l'égalité $D_{a b}=\omega(\sigma(a))$ du lemme 2.13 est bien sûr due au choix, arbitraire, de pointer la première lettre des images dans un germe canonique de substitution. Si $\sigma$ est une substitution non canonique par des motifs définie par des règles locales croissantes $D$ telle que max Supp $\sigma(a)=0$ pour toute lettre $a \in \mathcal{A}$, c'est-à-dire que les images des lettres par $\sigma$ sont pointées sur leur dernière lettre, alors on aura de façon symétrique $D_{a b}=\omega(\sigma(b))$.

\section{Caractérisation Des substitutions CANONiques AVEC RÈGLES LOCALES}

D'après le lemme 2.13, les règles locales croissantes sont une donnée fournie par le germe candidat à être une substitution. La question qui nous intéresse est de savoir quels sont les germes canoniques de substitutions pour lesquels ces règles locales en font des substitutions. Nous allons dans un premier temps considérer un cas particulièrement simple (paragraphe 3.1), celui des substitutions canoniques 
uniformes, c'est-à-dire celui où toutes les images de lettres ont le même support; c'est la généralisation aux substitutions par des motifs de la condition de longueur constante pour les substitutions usuelles, voir par exemple [6]. Puis nous introduirons au paragraphe 3.2 une distinction entre la nature des trous dans les images des lettres. Un premier type de germes canoniques admettant des règles locales peut alors être construit de manière naturelle en suivant l'image d'un attelage de wagons (paragraphe 3.3). Enfin nous donnons un théorème de caractérisation des germes canoniques de substitutions à règles locales au paragraphe 3.4.

\subsection{Substitutions PAR Des MOtifs Uniformes}

Il est en effet facile dans ce cas de donner une condition nécessaire et suffisante pour l'existence de règles locales qui se déduit aisément du lemme 2.13.

Proposition 3.1. Soit $\sigma$ un germe canonique de substitution uniforme sur $\mathcal{A}$. Soit $S$ le support commun à toutes les images des lettres. Il existe des règles locales croissantes qui font de $\sigma$ une substitution si et seulement si $S$ contient un et un seul élément de chaque classe de congruence modulo $|S|$. Ces règles sont alors : $D_{a b}=|S|$ pour tout couple de lettres $(a, b)$ dans $\mathcal{A}^{2}$.

Preuve. Ces conditions sont clairement suffisantes. La réciproque est une conséquence directe du lemme 2.13. On peut aussi voir directement que $D_{a a}=|S|$ sur ce cas simple : choisissons $a \in \mathcal{A}$ et considérons comme précédemment la réalisation $\left(n D_{a a}\right)_{n \in \mathbb{Z}}$ de $\sigma$ sur $a^{\mathbb{Z}}$; les $S+n D_{a a}, n \in \mathbb{Z}$, forment donc une partition de $\mathbb{Z}$, c'est-à-dire que l'ensemble $S$ contient un et un seul élément de chaque classe de congruence modulo $D_{a a}$; on a alors nécessairement $D_{a a}=|S|$.

Exemple 4. C'est par exemple le cas du germe de substitution $\sigma(a)=a \sqcup \sqcup b$ sur l'alphabet $\{a\}$ (considéré dans l'exemple 3 ); le cardinal de $S$ est 2, et on vérifie que les positions ( 0 et 3$)$ des lettres du motif forment un domaine fondamental modulo 2 .

Notons que cette caractérisation ne fait pas intervenir les valeurs attribuées aux éléments du support, donc en particulier l'alphabet $\mathcal{B}$.

\subsection{Trous du futur et du passé}

Supposons que $\sigma$ est une substitution canonique définie par des règles locales croissantes sur un alphabet $\mathcal{A}$, et $a$ une lettre de $\mathcal{A}$. Pour simplifier, on notera $S_{a}=\operatorname{Supp} \sigma(a), \omega_{a}=\omega(\sigma(a))=\left|S_{a}\right|$, et $l_{a}=l(\sigma(a))$. Considérons la réalisation $t$ de $\sigma$ sur $a^{\mathbb{Z}}$ conforme aux règles locales : on a nécessairement $t_{n}=n D_{a a}$, pour $n \in \mathbb{Z}$, d'après la définition 2.8 .

Définition 3.2. Supposons que $\sigma$ est une substitution canonique définie par des règles locales croissantes et soit $t$ la réalisation de $\sigma$ sur $a^{\mathbb{Z}}$ conforme aux règles locales. On appelle trou de $\sigma(a)$ les positions $k \notin S_{a}$ telles que $0=\min S_{a}<k<$ $\max S_{a}=l_{a}-1$. 
Les trous sont de deux types :

- les trous du passé sont remplis par des images selon la réalisation $t$ de lettres d'indice strictement négatif, ils sont de la forme $j+n D_{a a}$ où $j \in S_{a}$ et $n<0$;

- les trous du futur sont remplis par des images selon la réalisation $t$ de lettres d'indice strictement positif, ils sont de la forme $j+n D_{a a}$ où $j \in S_{a}$ et $n>0$.

\subsection{Attelages et wagons}

Nous présentons dans ce paragraphe une famille particulièrement simple de substitutions canoniques par des motifs avec règles locales. Considérons dans un premier temps l'exemple suivant :

Exemple 5. Soit $\sigma$ le germe canonique de substitution à règles locales sur l'alphabet $\{a\}$ défini par $\sigma(a)=a \sqcup \sqcup a \sqcup \sqcup a a a \sqcup a a \sqcup a a$ et ayant pour règle locale $D_{a a}=\omega_{a}=9$. Les trous du passé de $\sigma(a)$ sont situés aux indices 1, 2, 4 et 5 , tandis que les trous du futur sont situés aux indices 9 et 12 . On voit que, dans l'image du mot $a a$, les dernières lettres de l'image du premier $a$ doivent combler des trous du passé de l'image du second $a$, et que les premières lettres de l'image du second $a$ doivent, de façon complémentaire, combler des trous du futur de l'image du premier $a$.

On est alors conduit aux définitions suivantes :

Définition 3.3. Soit $\lambda \geq 2$ un entier. Un attelage de longueur $\lambda$ est une partition $\{P, F\}$ de l'intervalle $[0, \lambda-1]$ telle que $0 \in F$ et $\lambda-1 \in P$.

Définition 3.4. Un wagon de poids $\omega \geq \lambda$ et de longueur $l=\omega+\lambda$ portant l'attelage $\{P, F\}$ de longueur $\lambda$ est un motif pointé dont le support est

$$
F \cup[\lambda, \omega-1] \cup(P+\omega)=[0, l-1] \backslash(P \cup(F+\omega)) .
$$

La structure est simple à comprendre : chaque motif image d'une lettre est formé d'une partie centrale $[\lambda, \omega-1]$ de longueur arbitraire, encadrée par deux parties combinatoires qui s'engrènent l'une dans l'autre. La condition $\omega \geq \lambda$ est naturelle à étudier au vu de l'exemple 5, mais on verra au paragraphe 3.4 qu'elle peut être levée.

Exemple 6. Continuons l'exemple 5. Le motif $\sigma(a)$ est un wagon de poids $\omega=9$ et de longueur $l=15$ portant l'attelage $\{P, F\}$ de longueur $\lambda=6$ avec $F=\{0,3\}$ et $P=\{1,2,4,5\}$.

En assemblant des wagons compatibles entre eux, c'est-à-dire portant le même attelage, on construit une substitution canonique définie par des règles locales. Cependant, toutes ne sont pas obtenues ainsi, comme nous le verrons au paragraphe 3.4. 
Proposition 3.5. Soit $\sigma$ un germe canonique de substitution dont toutes les images de lettres $\sigma(a)$ sont des wagons (de longueurs et poids variés) portant le même attelage. Alors les règles locales $\left(D_{a b}\right)_{a, b \in \mathcal{A}}$, où $D_{a b}=\omega_{a}$ pour tout couple de lettres $(a, b)$, font de $\sigma$ une substitution.

Preuve. Soit $\sigma$ un germe canonique de substitution sur l'alphabet $\mathcal{A}$ dont toutes les images de lettres sont des wagons portant le même attelage $\{P, F\}$, de longueur $\lambda$. On considère une suite $x \in \mathcal{A}^{\mathbb{Z}}$, et on définit la suite d'entiers $t=\left(t_{n}\right)_{n \in \mathbb{Z}}$ par $t_{0}=0$ et $t_{n+1}-t_{n}=\omega_{x_{n}}$, pour tout $n \in \mathbb{Z}$. Il s'agit de montrer que $t$ est une réalisation de $\sigma$ sur $x$, c'est-à-dire que $\left\{S_{x_{n}}+t_{n} \mid n \in \mathbb{Z}\right\}$ forme une partition de $\mathbb{Z}$. Comme $\sigma\left(x_{n}\right)$ est un wagon d'attelage $\{P, F\}$, on a $S_{x_{n}}+t_{n}=A_{n} \cup B_{n} \cup C_{n}$, où $A_{n}=F+t_{n}$ (le demi-attelage arrière), $B_{n}=\left[\lambda+t_{n}, \omega_{x_{n}}-1+t_{n}\right]=\left[\lambda+t_{n}, t_{n+1}-1\right]$ (la caisse) et $C_{n}=P+\omega_{x_{n}}+t_{n}=P+t_{n+1}$ (le demi-attelage avant). Les ensembles $C_{n-1}$ et $A_{n}$ forment une partition de l'intervalle $\left[t_{n}, \lambda+t_{n}-1\right]$ (l'attelage, translaté de $t_{n}$ ), donc $C_{n-1} \cup A_{n} \cup B_{n}=\left[t_{n}, t_{n+1}-1\right]$ et on voit que $\bigcup_{n \in \mathbb{Z}}\left\{A_{n}, B_{n}, C_{n}\right\}$ est une partition de $\mathbb{Z}$.

On voit donc que les trous du passé du wagon $\sigma(a)$ sont les positions contenues dans $P$, tandis que les trous du futur sont les positions contenues dans $\left(F+\omega_{a}\right)$. Notons de plus que la condition $\omega_{a} \geq \lambda$ est équivalente à $\omega_{a} \geq \frac{l_{a}}{2}$, pour tout $a \in \mathcal{A}$ (on a $\left.l_{a}=\omega_{a}+\lambda\right)$.

Exemple 7. On reprend l'attelage défini par $\lambda=6, P=\{1,2,4,5\}, F=\{0,3\}$ de l'exemple 6 ; des wagons possibles sont : $\sigma(a)=a \sqcup \sqcup a \sqcup \sqcup b b b \sqcup c a \sqcup c a, \sigma(b)=$ $a \sqcup \sqcup b \sqcup \sqcup a \sqcup b a \sqcup b a, \sigma(c)=c \sqcup \sqcup a \sqcup \sqcup \sqcup a b \sqcup b a$. On a alors

$$
\sigma(a b c c a)=a \sqcup \sqcup a \sqcup \sqcup b b b a c a b c a a c b a a b a c a b a b a a a b a b a b b b \sqcup c a \sqcup c a .
$$

Notons que $\omega_{c}=\lambda$ : le wagon $\sigma(c)$ a une caisse vide, il est réduit à ses deux demi-attelages.

\subsection{THÉORÈME DE CARACTÉRISATION}

Les substitutions canoniques données par des wagons portant le même attelage sont un cas particulier : la famille de substitutions canoniques considérée dans le paragraphe précédent correspond au cas où le poids $\omega$ satisfait $\omega \geq \lambda$. Le cas général, résolu par le théorème suivant, correspond à ce type d'engendrement mais sans l'hypothèse $\omega \geq \lambda$.

Théorème 3.6. Un germe canonique de substitution $\sigma$ dont tous les motifs sont canoniques définit une substitution si et seulement si :

- soit toutes les images de lettres sont des mots;

- soit il existe un attelage $\{P, F\}$ de longueur $\lambda$ tel que toute lettre a admet une image par $\sigma$ de poids $\omega_{a}$, de longueur $l_{a}=\omega_{a}+\lambda$, et de support $\left[0, l_{a}-1\right] \backslash\left(P \cup\left(F+\omega_{a}\right)\right)$, où $\omega_{a}$ est un entier qui vérifie $P \cap\left(F+\omega_{a}\right)=\emptyset$.

Les règles locales croissantes sont alors $D_{a b}=\omega_{a}$ pour tout couple de lettres $(a, b) \in \mathcal{A}^{2}$. 
Preuve. Soit $\sigma$ une substitution canonique par des motifs avec règles locales croissantes sur $\mathcal{A}$. Par le lemme 2.13, ces règles locales sont $D_{a b}=\omega_{a}$ pour tout $(a, b) \in \mathcal{A}^{2}$. Supposons qu'il existe une lettre $a \in \mathcal{A}$ dont l'image admette des trous. Considérons la réalisation $t=\left(n \omega_{a}\right)_{n \in \mathbb{Z}}$ de $\sigma$ sur la suite $a^{\mathbb{Z}}$, et posons $E_{1}=\bigcup_{n<0}\left(S_{a}+n \omega_{a}\right)$ et $E_{2}=\bigcup_{n \geq 0}\left(S_{a}+n \omega_{a}\right)$. Alors $\left\{E_{1}, E_{2}\right\}$ est une partition de $\mathbb{Z}$.

Soit $\{P, F\}$ l'attelage défini par $P=\mathbb{N} \backslash E_{2}, \lambda=1+\max P$ et $F=[0, \lambda-1] \backslash P$. On a alors $E_{2}=\mathbb{N} \backslash P=F \cup[\lambda,+\infty[$.

Soit maintenant $b \in \mathcal{A}$ une lettre quelconque, et $t^{\prime}$ la réalisation de $\sigma$ sur $a^{\mathbb{Z}^{-}} \cdot b a^{\mathbb{Z}^{+}}$. Les règles locales impliquent que

$$
t^{\prime}=\left(\ldots,-3 \omega_{a},-2 \omega_{a},-\omega_{a}, 0, \omega_{b}, \omega_{b}+\omega_{a}, \omega_{b}+2 \omega_{a}, \ldots\right) .
$$

On a donc la partition de $\mathbb{Z}$ suivante : $\left\{E_{1}, S_{b}, E_{2}+\omega_{b}\right\}$, de sorte que

$$
S_{b}=E_{2} \backslash\left(E_{2}+\omega_{b}\right)=(\mathbb{N} \backslash P) \backslash\left(\left[\lambda+\omega_{b},+\infty\left[\cup\left(F+\omega_{b}\right)\right),\right.\right.
$$

soit $S_{b}=\left[0, \lambda+\omega_{b}-1\right] \backslash\left(P \cup\left(F+\omega_{b}\right)\right)$ comme annoncé; on vérifie aussi que $P \cap\left(F+\omega_{b}\right)=\emptyset$.

Réciproquement, si $\{P, F\}$ est un attelage de longueur $\lambda$ et $\sigma$ un germe canonique de substitution tel que pour toute lettre $a, S_{a}=\left[0, l_{a}-1\right] \backslash\left(P \cup\left(F+\omega_{a}\right)\right)$ et $P \cap\left(F+\omega_{a}\right)=\emptyset$, alors les règles locales $D_{a b}=\omega_{a}$ font de $\sigma$ une substitution. La preuve est similaire à celle de la proposition 3.5 : il faut montrer que $\left\{S_{x_{n}}+t_{n} \mid n \in \mathbb{Z}\right\}$ forme une partition de $\mathbb{Z}$, où cette fois

$$
S_{x_{n}}+t_{n}=\left[t_{n}, l_{x_{n}}-1+t_{n}\right] \backslash\left(\left(P+t_{n}\right) \cup\left(F+\omega_{x_{n}}+t_{n}\right)\right),
$$

soit $S_{x_{n}}+t_{n}=\left[t_{n}, t_{n+1}+\lambda-1\right] \backslash\left(\left(P+t_{n}\right) \cup\left(F+t_{n+1}\right)\right)$. Comme $\left\{P+t_{n+1}, F+t_{n+1}\right\}$ est une partition de $\left[t_{n+1}, t_{n+1}+\lambda-1\right]$, et $\left(P+t_{n}\right) \cap\left(F+t_{n+1}\right)=\emptyset$, on a $S_{x_{n}}+t_{n}=\left(\left[t_{n}, t_{n+1}-1\right] \cup\left(P+t_{n+1}\right)\right) \backslash\left(P+t_{n}\right)$, et ces ensembles forment bien une partition de $\mathbb{Z}$.

Exemple 8. Reprenons l'exemple 7. Nous allons introduire une lettre supplémentaire $d$ sans modifier les supports des autres lettres : on considère ainsi $\sigma(a)=a \sqcup \sqcup a \sqcup \sqcup b b b \sqcup c d \sqcup c d, \sigma(b)=a \sqcup \sqcup b \sqcup \sqcup a \sqcup b a \sqcup b a, \sigma(c)=c \sqcup \sqcup d \sqcup \sqcup \sqcup a b \sqcup b a$ et $\sigma(d)=a \sqcup \sqcup \sqcup \sqcup \sqcup \sqcup b c$. Dans l'image de $d$, les trous du passé sont en positions 1, 2, 4 et 5 comme pour les autres images de lettres, et les trous du futur en positions 3 et 6 . Le dernier trou du passé est au-delà du premier trou du futur. On a alors

$$
\sigma(a b d d c)=a \sqcup \sqcup a \sqcup \sqcup b b b a c d b c d a a b a a b a c b c d b c \sqcup a b \sqcup b a .
$$

Remarque 2. On voit que l'on n'a pas besoin de connaître les images des lettres pour savoir si la substitution est bien définie, il suffit de connaître les supports de ces images.

La longueur $\lambda$ de l'attelage associé à la substitution est le nombre de trous dans chaque motif; c'est un invariant de la substitution, indépendant de la lettre. 
Avec les notations du théorème 3.6 , dès que le poids $\omega_{a}$ de $\sigma(a)$ vérifie $\omega_{a} \geq \lambda$, la condition $P \cap\left(F+\omega_{a}\right)=\emptyset$ est automatiquement vérifiée, puisque les deux ensembles considérés sont contenus dans des intervalles disjoints; c'est le cas des wagons étudié ci-dessus, c'est-à-dire celui où il y a plus de lettres que de trous. Dans ce cas, il est facile de vérifier que tous les trous du passé précèdent les trous du futur, et qu'une image de lettre remplit tous les trous du passé de l'image de la lettre suivante, et tous les trous du futur de l'image de la lettre précédente.

Exemple 9. Notons que les indices $n$ dans la définition 3.2 des trous du passé et du futur peuvent être arbitrairement grands en valeur absolue. Considérons l'exemple suivant sur l'alphabet $\{a\}$ défini pour $k \geq 1: \sigma(a)=a \sqcup \ldots \sqcup a$, le nombre de trous étant égal à $2 k$. On a $D_{a a}=\omega_{a}=2$. Les trous du futur sont situés aux indices $2,4, \ldots, 2 k$ et sont remplis par les images des lettres d'indice $n=1, \ldots, k$ alors que les trous du passé sont aux indices $1,3, \ldots, 2 k-1$ et sont remplis par les images des lettres d'indice $n=-k, \ldots,-1$.

\subsection{Comment Reconnaître Si Un Germe Canonique De SUbSTitution DÉFINIT UNE SUBSTITUTION?}

Il n'est pas évident de reconnaître si un germe canonique de substitution est cohérent, c'est-à-dire si, muni des règles locales croissantes données par le lemme 2.13 , il définit bien une substitution : le problème est d'arriver à reconnaitre la nature des trous.

Un algorithme peut être donné comme suit : on se fixe une lettre $a$ et on considère le motif $\sigma(a)$; il est de poids $\omega_{a}$, donc il doit paver par translation par $\omega_{a}$. Chaque trou doit donc être congru modulo $\omega_{a}$ à un unique élément de $S_{a}$, par un vecteur de déplacement $n \omega_{a}$. Si $n>0$, il s'agit d'un trou du futur, et sinon, d'un trou du passé. On peut donc, d'abord, vérifier que le support du motif pave $\mathbb{Z}$ selon le vecteur de translation $\omega_{a}$ (sinon, la lettre à laquelle le trou est congru n'existe pas ou n'est pas unique); cette première étape a une complexité linéaire en $\omega_{a}$, donc en $l_{a}$. On peut ensuite reconnaître la nature des trous, et construire l'attelage $\{P, F\}$, ce qui est de complexité proportionnelle à la longueur $l_{a}$. Il suffit alors de vérifier que les autres motifs de la substitution correspondent au même attelage. On obtient alors un algorithme de complexité linéaire en la somme des longueurs des motifs.

\section{Points fixes Des substitutions par Des Motifs}

Un des intérêts de la notion classique de substitution est qu'elle permet d'engendrer des mots infinis qui se trouvent être invariants sous l'action de la substitution les engendrant. Le but de ce paragraphe est de définir pour les substitutions par des motifs une notion de point fixe engendré par substitution et de caractériser ces points fixes. Nous montrons en fait que ce sont des suites substitutives, c'est-àdire qu'on peut les engendrer par une substitution au sens classique, à un recodage près. 


\subsection{ENGENDREMENT DE POINTS FIXES}

Soit $\sigma$ une substitution (non canonique) par des motifs définie par des règles locales croissantes telle que

$-\mathcal{B}=\mathcal{A}$

- il existe $a \in \mathcal{A}$ tel que $\{-1,0,1\} \subset \operatorname{Supp} \sigma(a)$ et $\sigma(a)_{0}=a$.

On définit une suite de mots pointés $\left(w^{(k)}\right)$ comme suit :

- le premier terme est $w^{(0)}=\cdot a(\cdot a$ désigne le mot pointé de support $\{0\}$ dont l'unique lettre est $a$ );

- le terme $w^{(k)}$ étant connu, le terme suivant, $w^{(k+1)}$, est calculé en restreignant le motif pointé $\sigma\left(w^{(k)}\right)$ à la composante connexe de son support contenant 0 .

On vérifie que $[-k, k] \subset \operatorname{Supp} w^{(k)}$ et que $w^{(k)}$ est facteur de $w^{(k+1)}$. Ainsi la suite $\left(w^{(k)}\right)_{k \in \mathbb{N}}$ converge vers un mot biinfini $u$ qui est naturellement un point fixe de $\sigma$, c'est-à-dire que $\sigma(u)=u$. Ce mot $u$ est appelé point fixe engendré par la substitution en partant de $a$.

Cette définition s'étend au cas où il existe un mot pointé $w$ dont le support contient 0 et tel que $w$ est facteur de $\sigma(w)$, avec [min Supp $w-1, \max \operatorname{Supp} w+1] \subset$ Supp $\sigma(w)$. On pose alors $w^{(0)}=w$, et on définit $w^{(k)}$ puis $u$ comme ci-dessus.

Exemple 10. Soit $\sigma$ la substitution par des motifs uniformes définie par $\sigma(a)=$ $b \sqcup a \cdot a a \sqcup b$ et $\sigma(b)=a \sqcup b \cdot b b \sqcup a$. On a $w^{(0)}=\cdot a$, et donc $\sigma\left(w^{(0)}\right)=b \sqcup a \cdot a a \sqcup b$; on en déduit que $w^{(1)}=a \cdot a a$, et $\sigma\left(w^{(1)}\right)=b \sqcup a a a b b a \cdot a a b b a a a \sqcup b$, ce qui implique que $w^{(2)}=a a a b b a \cdot a a b b a a a$, et ainsi de suite. La substitution engendre donc le point fixe

$$
u=\ldots a a a b b b b a a b b b b a a a a b b a \cdot a a b b a a a a b b b b a a b b b b a a \ldots
$$

Remarque 3. Dans ce qui précède, il était possible de ne considérer que des germes canoniques de substitution sans perdre de généralité. L'existence d'images de lettres dont le support est différent du support du représentant canonique va jouer ici le rôle de « condition initiale». Le choix des supports des images des lettres est ici déterminant : des choix de supports différents dans une même classe donneront en général des points fixes distincts. Le théorème 3.6 ne s'applique $a$ priori qu'aux germes canoniques, mais le lemme 2.12 permet de se ramener à ce cas.

\subsection{Caractérisation}

Théorème 4.1. Soit $\sigma$ une substitution par des motifs définie par des règles locales croissantes, et soit u une suite biinfinie point fixe engendré par $\sigma$. Alors, u est une suite substitutive, c'est-à-dire qu'elle est l'image, par un morphisme lettre à lettre, du point fixe d'une substitution définie au sens classique par des mots. 
Preuve. Soit $t$ la réalisation de $\sigma$ sur $u$ conforme aux règles locales, de sorte que $u=\operatorname{Im}(\sigma, t)(u)$. Soit $\omega$ le plus petit poids des images des lettres, $\Omega$ le plus grand, $L_{1}=\min _{a \in \mathcal{A}} \min S_{a}$ et $L_{2}=1+\max _{a \in \mathcal{A}} \max S_{a}$. Nous supposons qu'il existe au moins un trou; dans le cas contraire, le résultat est immédiat. On a donc $\omega \geq 2$.

Considérons d'abord le mot fini pointé $w=u_{-N} \ldots u_{-1} \cdot u_{0} \ldots u_{N}$. Son image par $\sigma$ est un motif pointé $\sigma(w)$ facteur de $\sigma(u)=u$, dont le support contient l'intervalle $\left[-\omega(N+1)+L_{2}, \omega(N+1)+L_{1}-1\right]$. En effet, si $n<-N, t_{n} \leq-\omega(N+1)$ donc la dernière lettre du motif pointé $\sigma\left(u_{n}\right)+t_{n}$ est située à un indice strictement inférieur à $-\omega(N+1)+L_{2}$; de même, si $n>N$, la première lettre du motif pointé $\sigma\left(u_{n}\right)+t_{n}$ est située à un indice supérieur ou égal à $\omega(N+1)+L_{1}$.

Choisissons $N$ assez grand pour que $-\omega(N+1)+L_{2} \leq-N$ et $N+\Omega \leq \omega(N+$ 1) $+L_{1}$ (cela est possible car $\omega \geq 2$ ). L'intervalle $\left[-\omega(N+1)+L_{2}, \omega(N+1)+L_{1}-1\right]$ contient $[-N, N+\Omega-1]$, donc $[-N, N+\Omega-1] \subset \operatorname{Supp} \sigma(w)$. Plus généralement, pour tout $n \in \mathbb{Z},\left[t_{n}-N, t_{n}+N+\Omega-1\right]$ est contenu dans le support du motif pointé $\sigma\left(u_{n-N} \ldots u_{n-1} \cdot u_{n} \ldots u_{n+N}\right)+t_{n}$, qui est facteur de $u$. Ainsi, le mot (non pointé) de longueur $2 N+\Omega$ apparaissant dans $u$ aux positions $t_{n}-N$ à $t_{n}+N+\Omega-1$ ne dépend que du mot de longueur $2 N+1$ apparaissant dans $u$ aux positions $n-N$ à $n+N$.

On considère alors le nouvel alphabet $\widetilde{\mathcal{A}}=\mathcal{A}^{2 N+1}$ formé des mots de longueur $2 N+1$. Soit $\widetilde{u}$ la suite de $\widetilde{\mathcal{A}}^{\mathbb{Z}}$ obtenue en recodant $u$ de la manière suivante : pour tout $n \in \mathbb{Z}, \widetilde{u}_{n}=\left[u_{n-N} \ldots u_{n+N}\right]$. Soit $\widetilde{\sigma}$ la substitution (au sens classique) définie sur l'alphabet $\widetilde{\mathcal{A}}$ comme suit : l'image d'une lettre $\left[x_{-N} \ldots x_{N}\right]$ est le mot de longueur $\omega_{x_{0}}=\omega\left(\sigma\left(x_{0}\right)\right)$ défini par

$$
\widetilde{\sigma}\left(\left[x_{-N} \ldots x_{N}\right]\right)_{i}=\left[y_{i-N} \ldots y_{i+N}\right],
$$

où $0 \leq i<\omega_{x_{0}}$ et $y=\sigma\left(x_{-N} \ldots x_{-1} \cdot x_{0} \ldots x_{N}\right)$. On a alors, pour tout $n \in \mathbb{Z}$,

$$
\widetilde{\sigma}\left(\left[u_{n-N} \ldots u_{n+N}\right]\right)=\left[u_{t_{n}-N} \ldots u_{t_{n}+N}\right] \ldots\left[u_{t_{n+1}-1-N} \ldots u_{t_{n+1}-1+N}\right]
$$

(rappelons que $t_{n}+\omega_{u_{n}}=t_{n+1}$ ), soit $\widetilde{\sigma}\left(\widetilde{u}_{n}\right)=\widetilde{u}_{t_{n}} \widetilde{u}_{t_{n}+1} \ldots \widetilde{u}_{t_{n+1}-1}$, et on voit aisément que la suite $\widetilde{u}$ est point fixe de $\widetilde{\sigma}$. La suite $u$ est donc obtenue en appliquant le morphisme $\varphi: \widetilde{\mathcal{A}} \rightarrow \mathcal{A}$ qui associe à un mot de $\mathcal{A}^{2 N+1}$ sa lettre centrale, $\varphi\left(\left[x_{-N} \ldots x_{N}\right]\right)=x_{0}$. On a ainsi $u=\varphi(\widetilde{u})$.

Exemple 11. On considère le germe $\sigma$ suivant : $\sigma(1)=1 \cdot \sqcup 1 \sqcup 2$ et $\sigma(2)=1 \cdot \sqcup \sqcup 2$. On vérifie d'après le théorème 3.6 que le germe canonique associé $\sigma_{\mathrm{c}}$ muni des règles locales $D_{11}=D_{12}=3$ et $D_{21}=D_{22}=2$ réalise bien une substitution canonique, et on en déduit, comme $\min S_{1}=\min S_{2}$, que le germe $\sigma$ muni des mêmes règles locales réalise également une substitution.

En itérant $\sigma$ à partir du motif $w^{(0)}=1 \cdot 21$, comme décrit au paragraphe 4.1, on obtient une suite biinfinie $u$, point fixe engendré par $\sigma: \sigma(1 \cdot 21)=1 \sqcup 11 \cdot 2121 \sqcup 2$, donc $w^{(1)}=11 \cdot 2121, w^{(2)}=11211 \cdot 212112121$, etc. :

$$
u=\ldots 2121121121211211 \cdot 212112121121121211 \ldots
$$


La construction de la preuve du théorème 4.1 donne $\widetilde{\mathcal{A}}=\mathcal{A}^{3}, \widetilde{\sigma}([111])=\widetilde{\sigma}([112])=$ $\widetilde{\sigma}([211])=\widetilde{\sigma}([212])=[121][211][112]$, et $\widetilde{\sigma}([121])=\widetilde{\sigma}([122])=\widetilde{\sigma}([221])=\widetilde{\sigma}([222])=$ $[121][212]$ : la suite biinfinie

$$
\widetilde{u}=\ldots[211][112][121][211][112] \cdot[121][212][121][211][112] \ldots
$$

est bien point fixe de $\widetilde{\sigma}$.

On constate que $u$ est le point fixe engendré au sens usuel par la substitution $\tau: 1 \mapsto 211,2 \mapsto 21$, et qu'il admet les mêmes facteurs que le mot de Fibonacci, point fixe de la substitution $1 \mapsto 12,2 \mapsto 1$ (plus précisément, on obtient le mot de Fibonacci en tronquant $u$ : c'est $\left.\left(u_{n}\right)_{n \in \mathbb{Z}^{+}}\right)$.

Ici $u$ est point fixe d'une substitution au sens usuel, mais ce n'est pas toujours le cas. Dans l'exemple 10, le point fixe obtenu peut s'écrire (par la construction ci-dessus, suivie de l'identification des lettres de $\widetilde{\mathcal{A}}$ qui ont la même image par $\widetilde{\sigma}$ et $\operatorname{par} \varphi$ ) sous la forme $u=\varphi(\widetilde{u})$, où $\widetilde{u}$ est point fixe de la substitution $\widetilde{\sigma}$ sur $\left\{a, a^{\prime}, b, b^{\prime}\right\}$ définie par $\tilde{\sigma}(a)=a a^{\prime} b b^{\prime} a, \tilde{\sigma}\left(a^{\prime}\right)=a a a^{\prime} b b, \tilde{\sigma}(b)=b b^{\prime} a a^{\prime} b$ et $\tilde{\sigma}\left(b^{\prime}\right)=$ $b b b^{\prime} a a$, et $\varphi(a)=\varphi\left(a^{\prime}\right)=a, \varphi(b)=\varphi\left(b^{\prime}\right)=b$. Comme $\tilde{\sigma}$ est 5-uniforme, le mot $u$ est une suite 5 -automatique $[2,9]$. En revanche, il n'est point fixe d'aucune substitution sur $\{a, b\}$, uniforme ou non. En effet, $u$ contient les facteurs $a^{4}$ et $b^{4}$, et aucune autre puissance quatrième, or s'il était point fixe de $\psi$ il devrait contenir $\psi(a)^{4}$ et $\psi(b)^{4}$.

\section{Perspectives}

Les substitutions par des motifs ne permettent donc pas en dimension 1 de définir de nouvelles classes de mots infinis. Elles peuvent toutefois donner des définitions plus compactes de certains mots substitutifs. Pour progresser, il faut donc renoncer à certaines hypothèses : accepter des réalisations qui n'obéissent pas à des règles locales (donc trouver un moyen de donner une information sur l'agencement des motifs) ou considérer des substitutions $\sigma$ partielles qui ne sont définies que sur un ensemble $H$ de mots muni d'une structure convenable et avec $\sigma(H) \subset H$ pour permettre l'itération, et obtenir un point fixe.

La définition se transpose aisément en dimension supérieure mais alors un phénomène nouveau intervient : les seuls germes qui sont des substitutions sont uniformes. Cela vient du fait que contrairement à $\mathbb{Z}, \mathbb{Z}^{2}$ reste (discrètement) connexe quand on enlève un point, et donc que si on change une lettre dans un mot, la forme de son image ne peut pas changer. Il suffit en effet de considérer la suite constante $a^{\mathbb{Z}^{2}}$, et de remplacer la lettre en position 0 par une autre; on voit que les supports des motifs images des deux lettres doivent être les mêmes.

Remarquons qu'il existe par contre des substitutions partiellement définies en dimension 2 de support non constant qui ont des propriétés intéressantes, voir $[4,5]$. Ces substitutions sont basées sur le formalisme introduit dans [3] et qui, en dimension 1, permet d'associer à des substitutions dont la matrice d'incidence est 
inversible (la matrice d'incidence compte le nombre d'occurrences des lettres dans les images) des substitutions duales, qui ne sont rien d'autre que des substitutions par des motifs comme définies ici : ces substitutions engendrent alors des suites biinfinies points fixes qui sont des suites sturmiennes (pour plus de détails sur les suites sturmiennes, voir [14]); c'est ainsi que l'exemple 11 a été conçu.

\section{RÉFÉRENCES}

[1] B. Adamczewski, Y. Bugeaud and F. Luca, Sur la complexité des nombres algébriques. $C$. R. Acad. Sci. Paris Ser. I 339 (2004) 11-14.

[2] J.-P. Allouche and J. Shallit, Automatic sequences. Cambridge University Press (2003).

[3] P. Arnoux and S. Ito, Pisot substitutions and Rauzy fractals. Bull. Belg. Math. Soc. Simon Stevin 8 (2001) 181-207.

[4] P. Arnoux, V. Berthé and S. Ito, Discrete planes, $\mathbb{Z}^{2}$-actions, Jacobi-Perron algorithm and substitutions. Ann. Inst. Fourier (Grenoble) 52 (2002) 1001-1045.

[5] P. Arnoux, V. Berthé and A. Siegel, Two-dimensional iterated morphisms and discrete planes. Theoret. Comput. Sci. 319 (2004) 145-176.

[6] A. Cobham, Uniform tag sequences. Math. Syst. Theory 6 (1972) 164-192.

[7] F. Durand, Combinatorial and dynamical study of substitutions around the theorem of Cobham. Dynamics and Randomness, edited by A. Maass et al., Kluwer Academic Publishers (2002) 53-94.

[8] C. Goodman-Strauss, Matching rules and substitution tilings. Ann. Math. 147 (1998) $181-223$.

[9] F. von Haeseler, Automatic sequences, de Gruyter Expositions in Mathematics 36 (2003).

[10] C.W. Hansen, Dynamics of multi-dimensional substitutions. Ph.D. Thesis, George Washington University (2000).

[11] T. Kamae and L.Q. Zamboni, Maximal pattern complexity for discrete systems. Ergodic Theory Dynam. Systems 22 (2002) 1201-1214.

[12] T. Kamae and L.Q. Zamboni, Sequence entropy and the maximal pattern complexity of infinite words. Ergodic Theory Dynam. Systems 22 (2002) 1191-1199.

[13] J.C. Lagarias and Y. Wang, Tiling the line with translates of one tile. Invent. Math. 124 (1996) 341-365.

[14] M. Lothaire, Algebraic Combinatorics on words, Encyclopedia of Mathematics and its Applications 90, Cambridge University Press.

[15] M. Lothaire, Applied Combinatorics on words, Encyclopedia of Mathematics and its Applications 105, Cambridge University Press.

[16] A. Maes, An automata-theoretic decidability proof for first-order theory of $\langle\mathbf{N},\langle, P\rangle$ with morphic predicate P. J. Autom. Lang. Comb. 4 (1999), 229-245.

[17] A. Maes and M. Rigo, More on generalized automatic sequences. J. Autom. Lang. Comb. 7 (2002) 351-376.

[18] N. Pytheas Fogg, Substitutions in dynamics, arithmetics and combinatorics. Lect. Notes Math. 1794 (2002).

[19] M. Queffélec, Substitution dynamical systems. Spectral analysis. Lect. Notes Math. 1294 (1987).

[20] G. Rozenberg and A. Salomaa, The mathematical theory of L-systems. Academic Press (1980).

[21] D. Roy, Approximation to real numbers by cubic algebraic integers I. Proc. London Math. Soc. 88 (2004) 42-62. 
[22] D. Roy, Approximation to real numbers by cubic algebraic integers II. Ann. Math. 158 (2003) 1081-1087.

[23] O. Salon, Suites automatiques à multi-indices, Séminaire de Théorie des Nombres de Bordeaux, Exp. No. 4, Univ. Bordeaux-I (1986-1987) 4.01-4.27.

[24] O. Salon, Suites automatiques à multi-indices et algébricité. C. R. Acad. Sci. Paris Sér. I 305 (1987) 501-504.

[25] B. Solomyak, Dynamics of self-similar tilings. Ergodic Theory Dynam. Systems 17 (1997) 695-738.

[26] W.P. Thurston, Groups, tilings and finite state automata, Lectures notes distributed in conjunction with the Colloquium Series, AMS Colloquium lectures (1989).

Communiqué par J. Berstel.

Received 20 January, 2006. Accepted 3 January, 2007. 